\title{
A Rare Cause of Hemiparesis: Intracranial Mycotic Aneurysm-A Case Report, and Review of the Literature
}

\author{
Yao Christian Hugues Dokponou ${ }^{1,2^{*}}$, Mehdi Hakkou ${ }^{1,2}$, Olivier Ouambi ${ }^{1,2}$, \\ Nourou Dine Adeniran Bankole ${ }^{1,2}$, Abdessamad El Ouahabi, \\ ${ }^{1}$ Service de Neurochirurgie de l'Hôpital Des Spécialités, Rabat, Morocco \\ ${ }^{2}$ Faculté de Médecine et de Pharmacie, Université Mohammed V Rabat, Rabat, Morocco \\ Email: ^dokponou2407@gmail.com,drhakkou@yahoo.fr, dr.ouambi.lo@gmail.com, bankolenouroudine@yahoo.fr, \\ elouahabi.a@hotmail.fr
}

How to cite this paper: Dokponou, Y.C.H., Hakkou, M., Ouambi, O., Bankole, N.D.A. and El Ouahabi, A. (2021) A Rare Cause of Hemiparesis: Intracranial Mycotic Aneurysm-A Case Report, and Review of the Literature. Open Journal of Modern Neurosurgery, 11, 171-179. https://doi.org/10.4236/ojmn.2021.113021

Received: April 21, 2021

Accepted: July 12, 2021

Published: July 15, 2021

Copyright $\odot 2021$ by author(s) and Scientific Research Publishing Inc. This work is licensed under the Creative Commons Attribution International License (CC BY 4.0).

http://creativecommons.org/licenses/by/4.0/

\begin{abstract}
Background: The intracranial mycotic aneurysm is known to be a rare complication of infective endocarditis and it is more clinically challenging to get this diagnosis right when it happened to be in a patient without a past medical history of heart diseases. We report a documented case of mycotic aneurysm revealed by isolated left hemiparesis and our management with the collaboration of the cardiology department. Case Description: A 48-year-old male patient with a history of teeth loss, a chronic smoker presented with sudden heaviness in the left upper and lower limbs. No fever. Physical examination revealed a left hemiparesis of $3 / 5$ on the muscle tone scale without the stiffness of the neck. The CT-Scan and the MRI conclude of subarachnoid and cerebral hemorrhage with right temporal hematoma being most probably a vascular malformation. The cerebral arteriography concluded of a right Sylvian mycotic distal aneurysm in the M4 segment. Transesophageal echocardiography was performed and concluded of infectious endocarditis with mitral and aortic valvular disease grade II. Positive blood culture for staphylococcus coagulase-negative. The patient was managed with antibiotic therapy and clinically stable after 28 days. He was then transferred to the cardiology department for follow-up. Six (6) months later a CT-angiography was done for a check-up and shows no further changes in the aneurysm. The patient underwent surgery, two (2) months later, for clipping the aneurysm because the aneurysm did not regress in size. The aneurysm was then excluded with an eventless post-operative period, confirmed by controlled cerebral arteriography. The patient was discharged five (5) days later and he is doing well. Conclusion: Mycotic aneurysm is a rare consequence of infective endocardi-
\end{abstract}


tis. The distal sites of the middle cerebral artery are commonly found, and conservative treatment with a long course of antibiotics like amoxicillin 12 $\mathrm{g} / 24 \mathrm{~h}$ for 6 weeks or direct surgical clipping or excision can manage it.

\section{Keywords}

Mycotic Aneurysm, Endocarditis, Endovascular, Case Report

\section{Introduction}

Church (1869) was the first to build a relationship between intracranial mycotic aneurysm and endocarditis in a 13-year-old boy. The patient presented with left hemiparesis and subsequently died from a ruptured middle cerebral artery aneurysm [1]. A mycotic or infectious aneurysm is a dilation of an artery due to systemic seeding of infectious agent causing the damage of the vessel wall. Various organisms including predominantly bacterial can cause the aneurysm. It is a rare condition that is life-threatening. Delayed treatment can evolve to sepsis, spontaneous arterial rupture with significant bleeding and death. Twenty-five to thirty percent of patients with infective endocarditis would develop intracranial mycotic aneurysms [2]. They occur more frequently in the anterior circulation and may be multiple. Hemorrhage from an infectious aneurysm is a rare event but is associated with the poor neurological outcome with mortality approaching $80 \%$ in some studies [3].

A prolonged course of antibiotics is used, to treat mycotic aneurysm with conservative treatment if the patient presents intracranial hemorrhage, coma, severe comorbidities, stroke with severe damage. Surgery should be considered if the patient presents heart failure, uncontrolled infection, abscess, high embolic risk [4] [5] [6] [7] [8]. In this study, we report a positive outcome of conservative management of one case of mycotic aneurysm revealed by an isolated left hemiparesis mimicking intracranial tumor, followed by surgical treatment in a 48-year-old patient.

\section{Case Description}

Clinical: A 48-year-old man, a taxi driver, was admitted to the emergency department on the 10th of February 2020, complaining of sudden heaviness in the left upper and lower limbs. No fever, with a past medical history of teeth loss six weeks prior to his admission. The patient did not seek for any medical or dental treatment, he declared that his 42 and 44 teeth were affected with carries some months ago and fell off about six weeks prior to his admission. He is a chronic smoker about 120 packages of cigarettes per year for the past 25 years. The physical examination revealed a left hemiparesis of $3 / 5$ on the muscle tone scale without the stiffness of the neck. He was conscious with a GCS of 15, well oriented in time and space, and his family history was unremarkable. 
Diagnosis assessment: The MRI concludes of subarachnoid and cerebral hemorrhage with right parietal hematoma being most probably a vascular malformation or aneurysm (Figure 1). The angiography concluded with a right Sylvian mycotic distal aneurysm in the M4 segment (Figure 2). $\mathrm{CBC}$ and Ionogram are normal; CRP was $46 \mathrm{mg} / \mathrm{l}$ with antistreptolysin ASLO negative. Transesophageal echocardiography was performed and concluded of infectious endocarditis with mitral and aortic valvular disease grade II-III and mitral grade II aortic insufficiency (Figure 3). The blood culture was positive for staphylococcus coagulase-negative.

Management. The patient received amoxicillin $12 \mathrm{~g} / 24 \mathrm{~h}$ for 6 weeks. Our patient was not yet a candidate for cardiovascular surgery. After 28 days of treatment, we transferred him to the cardiology department to continue the management, decision-making, and follow-up.

Follow up: CT-angiography is carried out (3) three weeks later after conservative management, to check for the evolution of the mycotic aneurysm. Changes
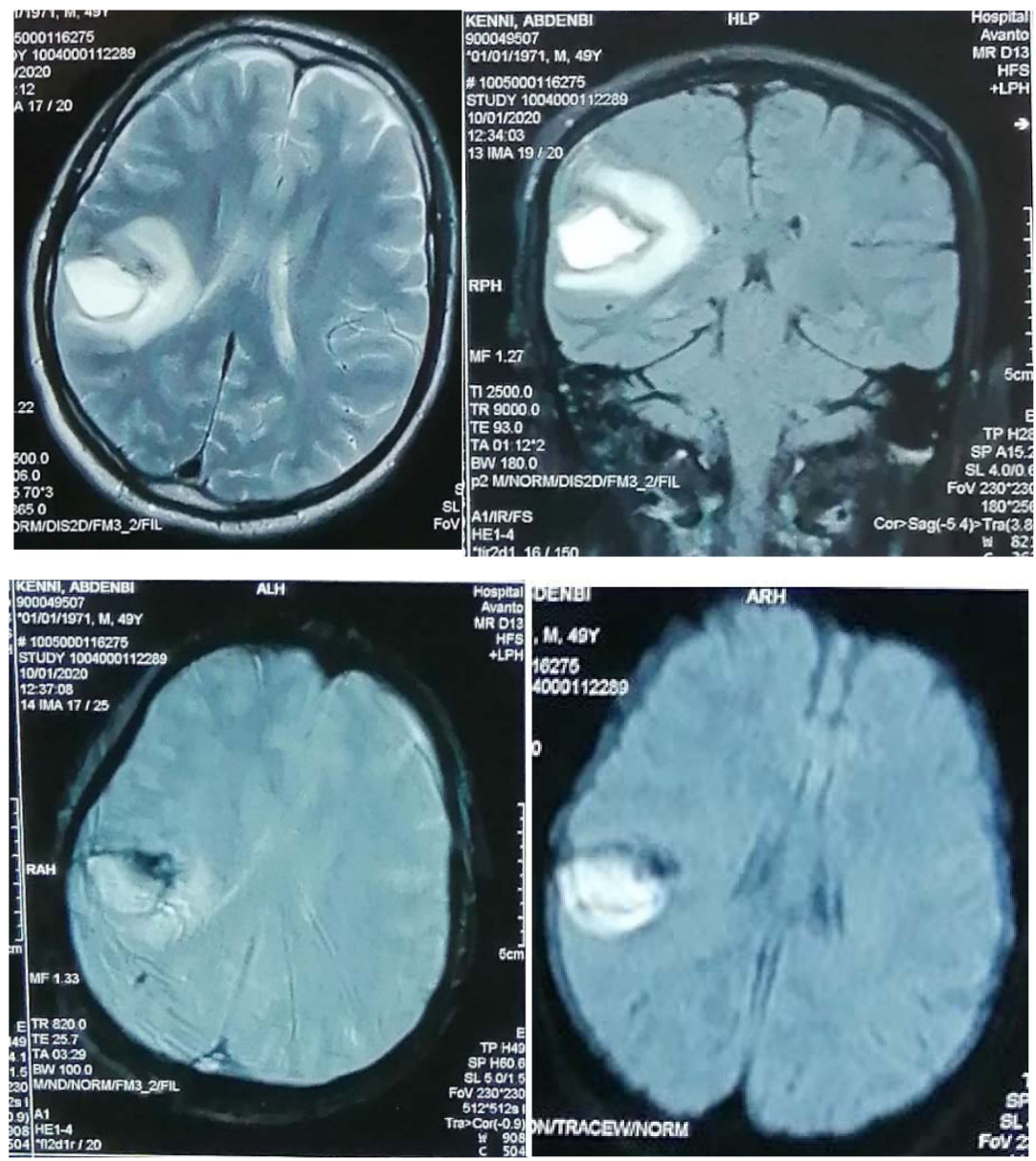

Figure 1. Axial MRI shows a right parietal cortical-sub-cortical lesion hyperintense on T2-weighted, flair, and diffusion surrounded by susceptibility appearing as low signal intensity due to blood on gradient-echo (GRE) images. A small rim of vasogenic edema surrounds the hematoma showing as hyperintense T2 and FLAIRE perilesional ring with mass effect. 


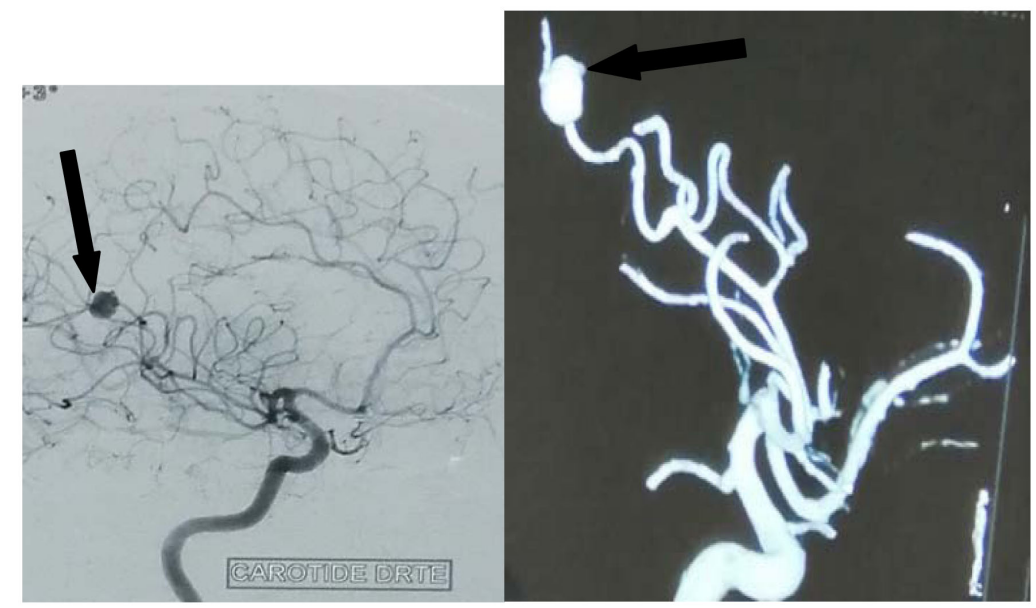

Figure 2. Arteriography. Right distal sylvian aneurysm at the M4 segment (black arrow), saccular in appearance, measuring $7.41 \mathrm{~mm} \times 6.42 \mathrm{~mm}$ with a relatively narrow neck measured at $2.82 \mathrm{~mm}$. Presence of an irregular aspect of the branches, with stenosis and dilation, as well as a felted aspect of the distal cortical branches: mycotic aneurysm.

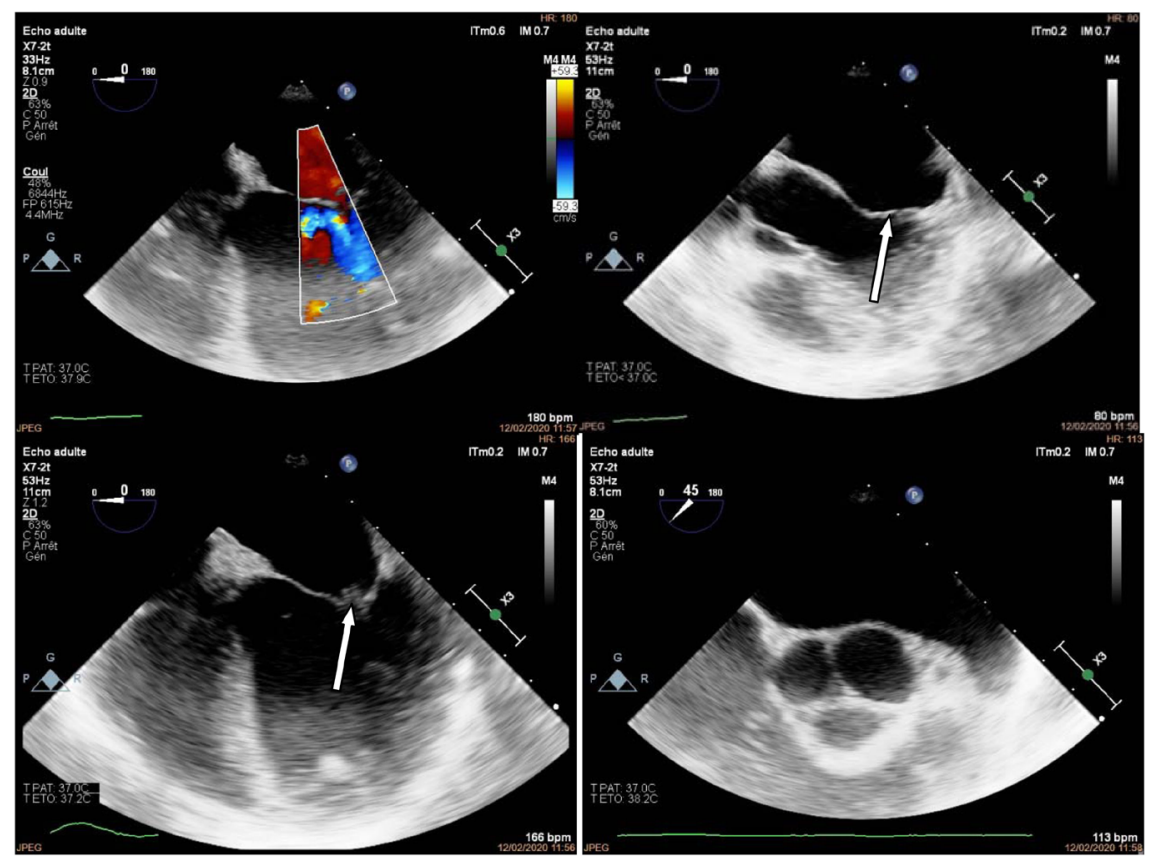

Figure 3. Infectious endocarditis on mitral and aortic valve disease with grade III aortic insufficiency and grade II mitral insufficiency (valvular vegetations = white arrow).

have been found neither in size nor in the shape of the aneurysm. Our patient was still clinically stable and repeated transoesophageal cardiac ultrasound was done. The valvular vegetations increased in size with the largest one passed from $12 \mathrm{~mm}$ to $17 \mathrm{~mm}$. The 6th-month check-up was done and found complete regression of mitral and aortic valvular vegetations followed by negative blood culture, confirming the healing of the patient from the infectious endocarditis. But, at the same time, the cerebral arteriography concluded a persistent right middle cerebral M4 mycotic aneurysm of the same size as it was six (6) months 
ago (Figure 4). Thereafter the patient was admitted to undergo surgery for clipping this aneurysm under neuronavigation since the aneurysm did not regress after medical treatment. A month later, the clipping of the Right M4 segment mycotic aneurysm by trans-sulcal approach was done without incidence (Figure 5). The patient was discharged five (5) days later with a confirmed exclusion of the mycotic aneurysm on the post-operative cerebral arteriography (Figure 6). He was doing well in the post-operative period.

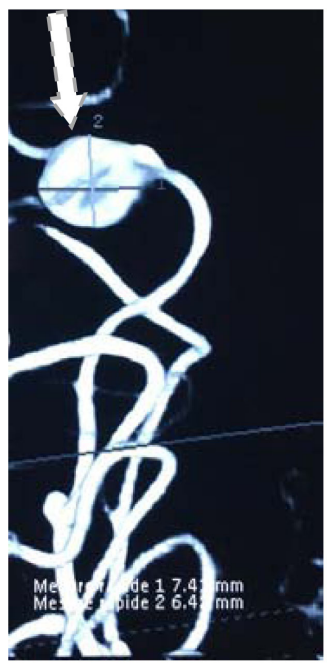

Figure 4. Cerebral arteriography at 6th month check up: Right distal sylvian aneurysm at the M4 segment (white arrow), saccular in appearance, measuring $7.41 \mathrm{~mm} \times 6.42 \mathrm{~mm} \times$ $2.84 \mathrm{~mm}$

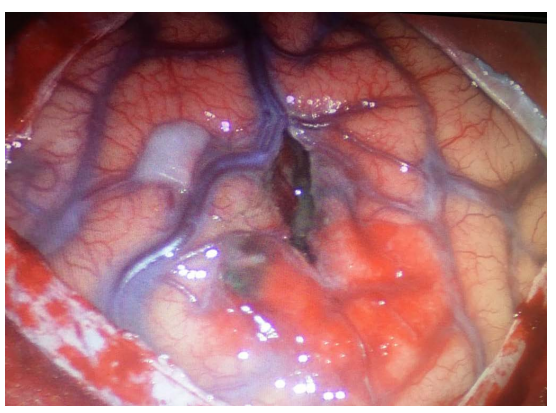

(a)

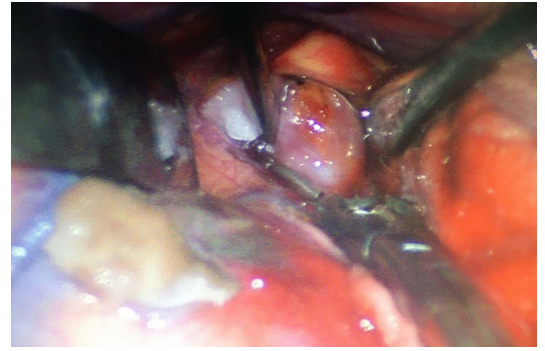

(b)

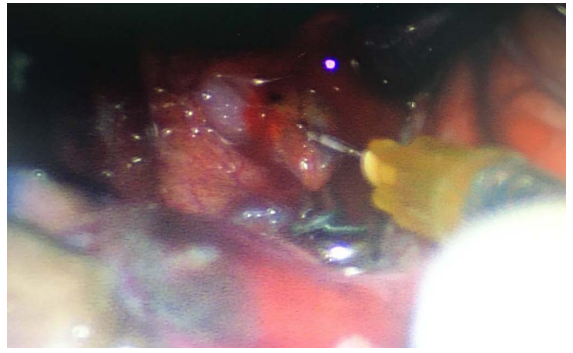

(c)

Figure 5. Clipping of the right M4 segment mycotic aneurysm by trans-sulcal approach. (a) Trans-sulcal approach; (b) The aneurysm has been dissected, exposed and clipped; (c) Aspiration of the aneurysm content with needle of insulin syringe. 


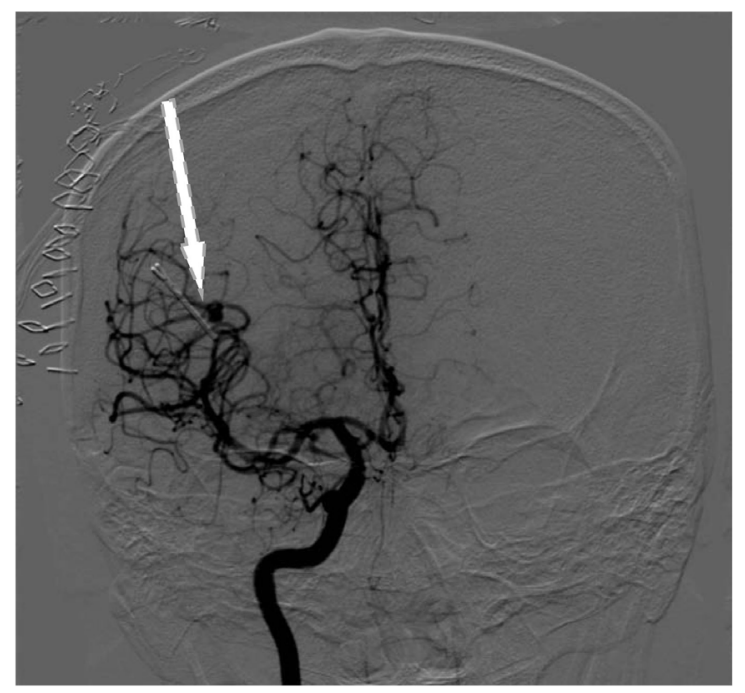

Figure 6. Right M4 segment mycotic aneurysm excluded by clipping (white arrow).

\section{Discussion}

Mycotic Aneurysm develops as a consequence of vessel wall necrosis following a showering of bacterial emboli into the circulation, usually due to bacterial endocarditis [9]. Their evolution is unpredictable even after the commencement of antibiotic therapy; they can either regress, develop de novo, or rupture. Variable outcomes have been reported, with a mortality rate from $16.8 \%$ [9] [10] to $83 \%$ [11]. The middle cerebral artery is the most frequent location of the infectious intracranial aneurysm in $63.5 \%$ of cases [11], which corresponds to our case report. Some authors advised surgery promptly for enlarging lesions and after a course of antibiotics for lesions that did not regress in size. We have got the same finding in our case; with the 3rd-week cerebral angiography showing no changes in the size of the lesion. Although we do not promptly go into surgery to excise the intracranial aneurysm, we firstly went on with the conservative treatment option. In fact, Kannoth et al. were not able to show any benefits of early or late surgical treatment, whereas Bohmfalk et al. hypothesized that an intervention in the acute phase of the disease is associated with an increased intraoperative risk for aneurysm rupture.

After a thorough discussion with our colleague of the cardiovascular department, transoesophageal cardiac ultrasound was needed to appreciate the evolution of the valvular vegetation in size. The valvular vegetations increased in size with the largest one passed from $12 \mathrm{~mm}$ to $17 \mathrm{~mm}$; shifting the patient for cardiosurgery indication [11] [12]. On the second hand, Neurosurgery or endovascular therapy is recommended for very large, enlarging, or ruptured intracranial infectious aneurysms; or after a silent embolism or transient ischaemic attack. Cardiac surgery, if indicated, is recommended without delay. Our patients do not present any of those complications and cannot be managed as such. Moreover, conservative treatment and monitoring are recommended for inpatient without heart failure, uncontrolled infection, abscess, high embolic risk [13] 
[14]. This is what, Lee et al. described in their studies by showing an increased morbidity and mortality rate in patients with intervention after antibiotics because of fulminant septicemias and hemorrhages. Therefore, the timing of the operative intervention should be determined after taking the clinical situation of the patient into account. The benefit of antibiotics treatment course over surgical clipping or excision of the mycotic aneurysm has not been thoroughly studied and need be for meta-analysis to bring about diagram for therapeutic decision making, knowing that in the literature there is no consensus on when and which patient fit for a prolonged course of antibiotics vs surgical treatment or both. Hamisch CA et al. described both conservative and surgical management of mycotic aneurysm but were not able to conclude on a benefit of one method over the other. The patient of our case report is doing well after the conservative treatment and the aneurysm does not increase nor decreased in size on the 6th-month cerebral arteriography; he has then undergone surgery thereafter for aneurysm clipping. We believe the surgical exclusion of mycotic aneurysm should be done in all patients just after the recommended six (6) weeks antibiotic course with evidence of the patient completely healing from infective endocarditis and if there is no evidence that the aneurysm is regressing under medical treatment.

\section{Conclusion}

Mycotic aneurysm, a rare consequence of infectious endocarditis, the distal sites of middle cerebral artery commonly found, and conservative treatment with a long course of antibiotics like amoxicillin $12 \mathrm{~g} / 24 \mathrm{~h}$ for 6 weeks and/or direct surgical clipping to exclude the aneurysm can best manage it. Various results are found in the Literature. There is no consensus, so far, on which method brings about a better outcome; some authors advocate both. Multimodal meta-analysis is necessary to conclude on the real benefit of early surgery in the case of mycotic aneurysm.

\section{Conflicts of Interest}

The authors declare not having any conflict of interest in this case report and there are no financial resources.

\section{Author Contributions}

Yao Christian Hugues Dokponou: Conceptualization, Writing original draft, \& editing. Mehdi HAKKOU: Writing_review \& editing. Olivier OUAMBI: Writing-review \& editing. Nourou Dine Adeniran BANKOLE: Writing-review \& editing. Abdessamad El Ouahabi: Supervision, Validation, \& review.

\section{Ethics and Reporting Guidelines}

Informed consent and verbal permission were obtained from the patient prior to the submission of this article. Also, this article respects both the Consensus-based 
Clinical Case Reporting Guideline and the Recommendations for the Conducting, Reporting, Editing, and Publication of Scholarly Work in Medical Journals [15] [16].

\section{References}

[1] Phuong, L.K., Link, M. and Wijdicks, E. (2002) Management of Intracranial Infectious Aneurysms: A Series of 16 Cases. Neurosurgery, 51, 1145-1151. https://doi.org/10.1097/00006123-200211000-00008

[2] Chapot, R., Houdart, E., Saint-Maurice, J.-P., Aymard, A., Mounayer, C., Lot, G., et al. (2002) Endovascular Treatment of Cerebral Mycotic Aneurysms. Radiology, 222, 389-396. https://doi.org/10.1148/radiol.2222010432

[3] Hamisch, C.A., Mpotsaris, A., Timmer, M., Reiner, M., Stavrinou, P., Brinker, G., et al. (2016) Interdisciplinary Treatment of Intracranial Infectious Aneurysms. Cerebrovascular Diseases, 42, 493-505. https://doi.org/10.1159/000448406

[4] Habib, G., Lancellotti, P., Antunes, M.J., Bongiorni, M.G., Casalta, J.-P., Del Zotti, F., et al. (2015) 2015 ESC Guidelines for the Management of Infective Endocarditis: The Task Force for the Management of Infective Endocarditis of the European Society of Cardiology (ESC) Endorsed by: European Association for Cardio-Thoracic Surgery (EACTS), the European Association of Nuclear Medicine (EANM). European Heart Journal, 36, 3075-3128. https://doi.org/10.1093/eurheartj/ehv319

[5] Brust, J.C.M., Dickinson, P.C.T., Hughes, J.E.O. and Holtzman, R.N.N. (1990) The Diagnosis and Treatment of Cerebral Mycotic Aneurysms. Annals of Neurology, 27, 238-346. https://doi.org/10.1002/ana.410270305

[6] Thuny, F., Grisoli, D., Collart, F., Habib, G. and Raoult, D. (2012) Management of Infective Endocarditis: Challenges and Perspectives. Lancet, 379, 965-975. https://doi.org/10.1016/S0140-6736(11)60755-1

[7] Kannoth, S., Iyer, R., Thomas, S.V., Furtado, S.V., Rajesh, B.J., Kesavadas, C., Radhakrishnan, V.V. and Sarma, P.S. (2007) Intracranial Infectious Aneurysm: Presentation, Management and Outcome. Journal of the Neurological Sciences, 256, 3-9. https://doi.org/10.1016/j.jns.2007.01.044

[8] Habib, G. (2006) Management of Infective Endocarditis. Heart, 92, 124-130. https://doi.org/10.1136/hrt.2005.063719

[9] Regelsberger, J., Elsayed, A., Matschke, J., Lindop, G., Grzyska, U., van den Boom, L. and Venne, D. (2011) Diagnostic and Therapeutic Considerations for 'Mycotic' Cerebral Aneurysms: 2 Case Reports and Review of the Literature. Central European Neurosurgery, 72, 138-143. https://doi.org/10.1055/s-0031-1279756

[10] Bohmfalk, G.L., Story, J.L., Wissinger, J.P. and Brown Jr:, W.E. (1978) Bacterial Intracranial Aneurysm. Journal of Neurosurgery, 48, 369-382. https://doi.org/10.3171/jns.1978.48.3.0369

[11] Horstkotte, D., Follath, F., Gutschik, E., Lengyel, M., Oto, A., Pavie, A., Soler-Soler, J., Thiene, G., von Graevenitz, A., Priori, S.G., Garcia, M.A., Blanc, J.J., Budaj, A., Cowie, M., Dean, V., Deckers, J., Fernandez, B.E., Lekakis, J., Lindahl, B., Mazzotta, G., Morais, J., Oto, A., Smiseth, O.A., Lekakis, J., Vahanian, A., Delahaye, F,. Parkhomenko, A., Filipatos, G., Aldershvile, J. and Vardas, P. (2004) Guidelines on Prevention, Diagnosis and Treatment of Infective Endocarditis Executive Summary: The Task Force on Infective Endocarditis of the European Society of Cardiology. European Heart Journal, 25, 267-276. https://doi.org/10.1016/j.ehj.2003.11.008

[12] Utoh, J., Miyauchi, Y., Goto, H., Obayashi, H. and Hirata, T. (1995) Endovascular Approach for an Intracranial Mycotic Aneurysm Associated with Infective Endo- 
carditis. The Journal of Thoracic and Cardiovascular Surgery, 110, 557-559. https://doi.org/10.1016/S0022-5223(95)70259-8

[13] Zanaty, M., Chalouhi, N., Starke, R.M., Tjoumakaris, S., Gonzalez, L.F., Hasan, D., Rosenwasser, R. and Jabbour, P. (2013) Endovascular Treatment of Cerebral Mycotic Aneurysm: A Review of the Literature and Single Center Experience. BioMed Research International, 2013, Article ID: 151643.

https://doi.org/10.1155/2013/151643

[14] Naber, C.K., Erbel, R., Baddour, L.M. and Horstkotte, D. (2007) New Guidelines for Infective Endocarditis: A Call for Collaborative Research. International Journal of Antimicrobial Agents, 29, 615-616.

https://doi.org/10.1016/j.ijantimicag.2007.01.016

[15] Gagnier, J.J., Kienle, G., Altman, D.G., Moher, D., Sox, H., Riley, D., et al. (2013) The CARE Guidelines: Consensus-Based Clinical Case Reporting Guideline Development. Global Advances in Health and Medicine, 2, 38-43. https://doi.org/10.1136/bcr-2013-201554

[16] International Committee of Medical Journal Editors (2020) Recommendations for the Conducting, Reporting, Editing, and Publication of Scholarly Work in Medical Journals. ICMJE 2019. http://www.icmje.org/recommendations/ 